Review Article

\title{
Efficacy of Terpenoid in Attenuating Aortic Atherosclerosis in Apolipoprotein-E Deficient Mice: A Meta-Analysis of Animal Studies
}

\author{
Han Liu $\mathbb{D}^{1},{ }^{1}$ Yang Zhang, ${ }^{2}$ Siqiao Sun, ${ }^{2}$ and Shuai Wang $\mathbb{D}^{2}$ \\ ${ }^{1}$ Department of Respiration, The First Hospital of Jilin University, Changchun, Jilin, China \\ ${ }^{2}$ Department of Vascular Surgery, The First Hospital of Jilin University, Changchun, Jilin, China \\ Correspondence should be addressed to Shuai Wang; wang_shuai@jlu.edu.cn
}

Received 29 April 2019; Revised 10 June 2019; Accepted 17 June 2019; Published 17 July 2019

Academic Editor: Ahmed Abdel-Latif

Copyright (C) 2019 Han Liu et al. This is an open access article distributed under the Creative Commons Attribution License, which permits unrestricted use, distribution, and reproduction in any medium, provided the original work is properly cited.

Background. The apolipoprotein E knockout (ApoE -/-) mouse model is well established for the study of terpenoids in the prevention of atherosclerosis. Studies investigating the clinical benefit of terpenoids in humans are scarce. This systematic review and metaanalysis evaluated the effects of terpenoid administration on atherosclerotic lesion area in ApoE -/- mice. Methods. A comprehensive literature search using PubMed, Embase, and the Cochrane Library databases was performed to identify studies that assessed the effects of terpenoids on atherosclerosis in ApoE -/- mice. The primary outcome was atherosclerotic lesion area, and study quality was estimated using SYRCLE's risk of bias tool. Results. The meta-analysis included 25 studies. Overall, terpenoids significantly reduced atherosclerotic lesion area when compared to vehicle control $(P<0.00001$; SMD: $-0.55 ; 95 \%$ CI: $-0.72,-0.39)$. In terpenoid type and dose subgroup analyses, sesquiterpenoid $(P=0.002$; SMD -0.93; 95\% CI: $-1.52,-0.34)$, diterpenoid $(P=0.01$; SMD: -0.30 ; 95\% CI: 0.54, -0.06), triterpenoid ( $P<0.00001$; SMD: -0.66 ; 95\% CI: -0.94, -0.39$)$, tetraterpenoid $(P<0.0001$; SMD: -1.81 ; 95\% CI: $-2.70,-0.91)$, low dose $(P=0.0001$; SMD: $-0.51 ; 95 \%$ CI: $-0.76,-0.25)$, medium dose $(P<0.0001$; SMD: $-0.48 ; 95 \%$ CI: $-0.72,-0.24)$, and high dose $(P=0.002$; SMD: $-1.07 ; 95 \%$ CI: $-1.74,-0.40)$ significantly decreased atherosclerotic lesion area when compared to vehicle control. PROSPERO register number is CRD42019121176. Conclusion. Sesquiterpenoid, diterpenoid, triterpenoid, and tetraterpenoid have potential as antiatherosclerotic agents with a wide range of doses. This systematic review provides a reference for research programs aimed at the development of terpenoid-based clinical drugs.

\section{Introduction}

Atherosclerosis is the main cause of cardiovascular disease, which is the leading cause of death globally $[1,2]$. Dyslipidemia and oxidative stress are relevant to the pathogenesis of atherosclerosis [3, 4]. Therefore, statin-based lipid-modifying therapies, such as atorvastatin and rosuvastatin, are effective for lowering blood cholesterol levels and providing clinical benefits in patients with cardiovascular disease. However, the morbidity and mortality associated with atherosclerosis remain high [5], and there is an urgent unmet clinical need for novel prevention and treatment strategies [6].

In recent years, studies have shown that natural compounds, such as flavonoids, alkaloids, and terpenoids, attenuate atherosclerosis [7-9]. Terpenoids are a large and diverse class of naturally occurring organic chemicals that are similar to terpenes. Most terpenoids are multicyclic structures with oxygen-containing functional groups. Furthermore, terpenoids have a wide range of pharmacological effects, including antitumor, anti-inflammatory, antiatherosclerotic, and antimalarial activities [10-12]. The majority of studies on the antiatherosclerotic effects of terpenoids have focused on paclitaxel, $[13,14]$ which is a natural diterpene, and consensus on the antiatherosclerotic effects of other terpenoids has not been reached.

Studies in animals allow for initial investigations on the safety and efficacy of new interventions and provide an important link between basic research and clinical trials. The apolipoprotein E knockout (ApoE -/-) mouse model spontaneously develops atherosclerotic plaques and 
is commonly used to mimic the pathophysiological process of atherosclerosis in humans $[15,16]$. The present systematic review and meta-analysis evaluated the effects of terpenoid administration on atherosclerotic lesion area in ApoE -/mice, in an effort to understand the clinical potential of terpenoids as antiatherosclerotic agents.

\section{Materials and Methods}

2.1. Reporting Standards. This systematic review complies with the Preferred Reporting Items for Systematic Reviews and Meta-Analyses (PRISMA) statement. The systematic review protocol was prepared using the SYRCLE format for animal intervention studies $[17,18]$.

2.2. Search Strategy. An experienced information specialist (HL) searched the PubMed, Embase, and Cochrane Library databases from January 2001 to December 2018 using the keywords: "atherosclerosis," "atherogenesis," "apolipoproteins e," "apoe," "mice," and "terpenoid" and the following search strategies: (atherosclerosis OR atherogenesis) AND ("apolipoprotein $* \mathrm{e}$ " OR apoe) AND (mice OR mouse) AND (terpenoid OR hemiterpenoid OR monoterpenoid OR sesquiterpenoid OR diterpenoid OR sesterterpenoid OR triterpenoid OR tetraterpenoid OR polyterpenoid). The reference lists of included and review articles were manually searched to identify additional relevant studies. The search was performed on December 10, 2018, and was restricted to articles published in the English language.

2.3. Inclusion and Exclusion Criteria. Inclusion criteria are as follows: (1) study design: original research; (2) animal model: ApoE -/- mice; (3) disease model: atherosclerosis; (4) intervention: terpenoids. Exclusion criteria are as follows: (1) case reports, conference abstracts, review articles, and editorials, (2) missing data, or (3) overlapping or duplicate datasets.

2.4. Study Selection. Two reviewers (YZ and SS) independently examined the titles and abstracts of the articles identified by the literature search to select eligible studies. The full text of potentially relevant articles was retrieved and independently examined by two reviewers (YZ and SW) to determine whether these studies met the inclusion criteria. Disagreements on study selection were resolved by discussion and consensus.

2.5. Data Extraction. Two reviewers (HL and SW) independently extracted data from eligible studies, including the first author's name, publication year, age of mice, gender, diet, terpenoid dose, duration and route of treatment, control and treatment group sample sizes, location of the atherosclerotic lesion, stain used to assess the atherosclerotic lesion, and atherosclerotic lesion area. Data that were presented graphically in the original publications were extracted using Adobe Photoshop 7.0.

The primary outcome was atherosclerotic lesion area measured as a percentage or a numerical value.
Disagreements on data extraction were resolved by discussion and consensus.

2.6. Quality Assessment. Two investigators (SS and SW) independently assessed the quality of the included studies using SYRCLE's risk of bias tool, which contains domains evaluating sequence generation, baseline characteristics, allocation concealment, random housing, blinding, random and selective outcome assessments, incomplete outcomes data, and other sources of bias [44]. Publication bias was detected by visual inspection of funnel plots.

Disagreements on quality assessment were resolved by discussion and consensus.

2.7. Data Synthesis and Statistical Analysis. Statistical analyses were performed using Review Manager (RevMan Version 5.3 for Windows Copenhagen: The Nordic Cochrane Centre, The Cochrane Collaboration, 2014). Standardized mean differences (SMD) with 95\% confidence intervals (CI) were calculated to reflect the effects of terpenoids or vehicle control on atherosclerotic lesion area. A random-effects model was used to pool studies. Heterogeneity was determined as moderate $\left(I^{2} \geq 30 \%\right)$ or high $\left(I^{2} \geq 50 \%\right)$ using the inconsistency index.

Multiple independent groups in a study (e.g., different terpenoid doses) were considered separate datasets. In eleven studies $[19,20,24,26,28,33,34,38-40,42]$, multiple groups that tested different terpenoid doses were compared to a single control group. In order to avoid an artificial increase in sample size in the pooled analysis, the number of animals in the control group for each study was divided by the number of comparator groups.

Subanalyses were conducted to investigate the effects of sesquiterpenoid, diterpenoid, triterpenoid, and tetraterpenoid on the atherosclerotic lesion area.

Sensitivity analyses were conducted to determine whether the findings were robust. $P<0.05$ was considered statistically significant.

\section{Results}

3.1. Study Selection. The search identified 1,032 articles. Titles and abstracts were screened, and 40 studies were considered potentially eligible for inclusion. After evaluating full-text articles, nine studies were excluded, because outcomes data were not reported [45-53], and six studies were excluded, because multiple interventions were assessed [54-59]. Finally, 25 studies were included in the present meta-analysis [19-43] (Figure 1).

3.2. Study Characteristics. The characteristics of the 25 included studies are described in Table 1. These studies provided 59 datasets and involved 707 animals.

Three studies used sesquiterpenoid as the intervention [19-21], nine studies used diterpenoid as the intervention [22-30], ten studies used triterpenoid as the intervention [31$40]$, and three studies used tetraterpenoid as the intervention [41-43]. 


\begin{tabular}{|c|c|c|c|c|c|c|c|c|}
\hline 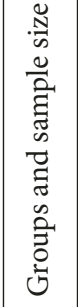 & 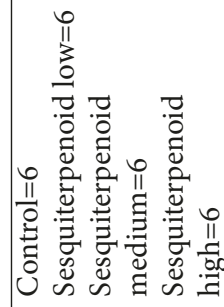 & 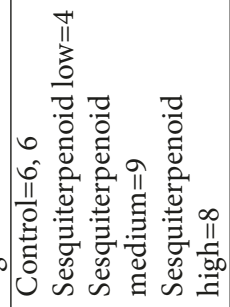 & 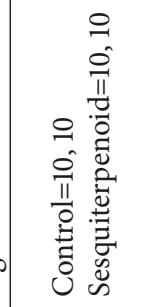 & 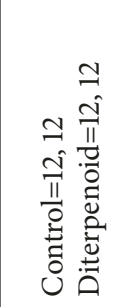 & 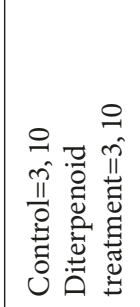 & 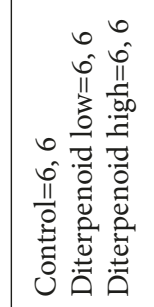 & 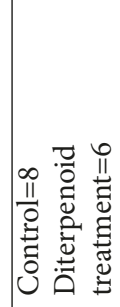 & 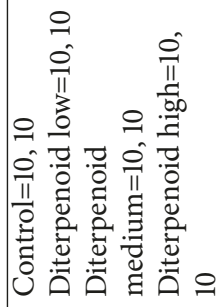 \\
\hline 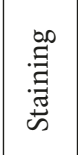 & 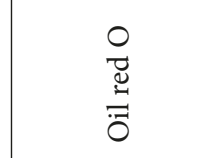 & $\begin{array}{l}\stackrel{0}{\overrightarrow{0}} \\
\stackrel{\Xi}{\overrightarrow{0}}\end{array}$ & $\begin{array}{l}0 \\
\stackrel{0}{\Xi} \\
\stackrel{⿹}{0}\end{array}$ & 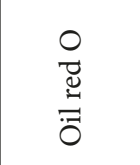 & 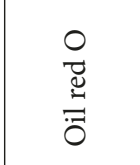 & $\begin{array}{l}0 \\
\stackrel{0}{\Xi} \\
\stackrel{\Xi}{0}\end{array}$ & 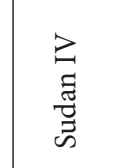 & 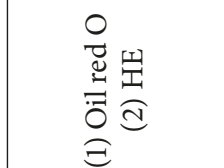 \\
\hline 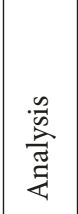 & 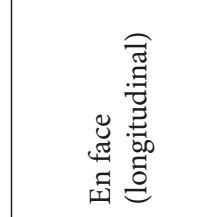 & 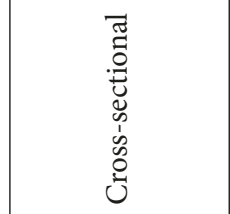 & 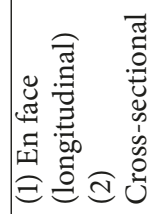 & 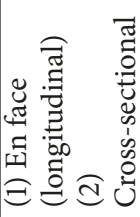 & 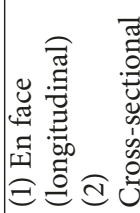 & 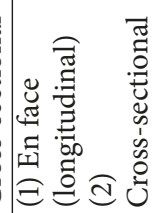 & 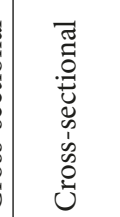 & 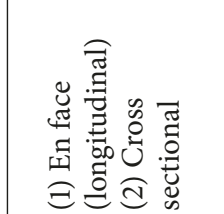 \\
\hline 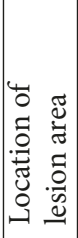 & 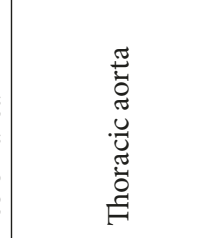 & 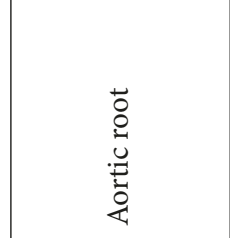 & 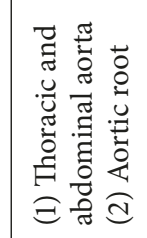 & 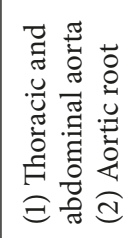 & 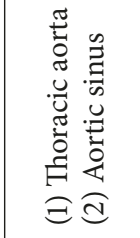 & 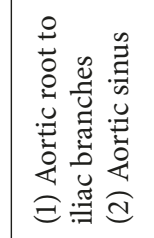 & 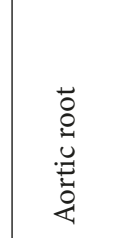 & 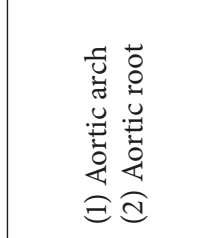 \\
\hline $\begin{array}{l}\stackrel{0}{0} \\
\check{a}\end{array}$ & بُ. & $\stackrel{\dot{f}}{.}$ & $\circlearrowleft$ & $\circlearrowleft$ & $\circlearrowleft$ & $\circlearrowleft$ & $\circlearrowleft$ & 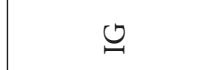 \\
\hline $\begin{array}{l}\mathscr{ٌ} \\
\stackrel{\circ}{\circ}\end{array}$ & 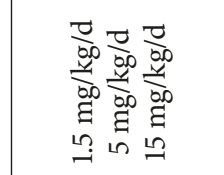 & 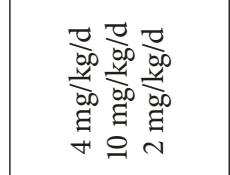 & 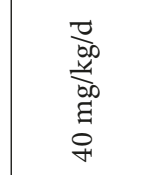 & 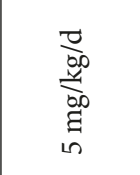 & 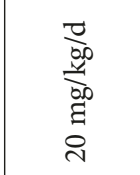 & 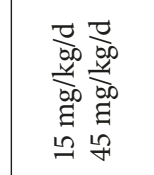 & 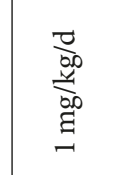 & 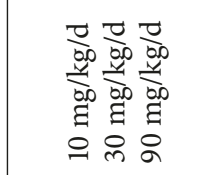 \\
\hline 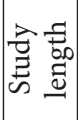 & $\underset{\sim}{\stackrel{3}{~}}$ & 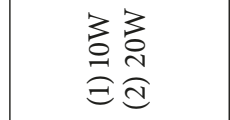 & 莕 & 3 & 3 & 芆 & 3 & 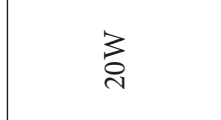 \\
\hline$\stackrel{\vec{\nu}}{.}$ & 䚾 & 旺 & 䚾 & 旺 & 旺 & 䚾 & 呈 & Оి \\
\hline ॐ & $\sim$ & $\frac{\pi}{\Sigma \pi}$ & 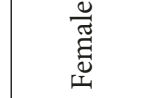 & $\frac{\pi}{\tilde{\pi}^{\pi}}$ & $\frac{\ddot{\pi}}{\Sigma^{\pi}}$ & $\frac{\ddot{\pi}}{\Sigma}$ & $\frac{0}{\tilde{\pi}^{\frac{\pi}{z}}}$ & $\frac{\ddot{\pi}}{\Sigma}$ \\
\hline 桇 & 3 & $\underset{\exists}{3}$ & $3_{\infty}^{3}$ & 3 & $3_{\infty}^{3}$ & 3 & 芆 & 3 \\
\hline 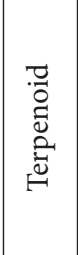 & 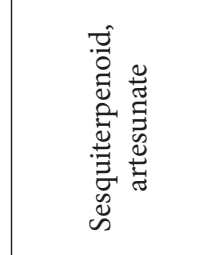 & 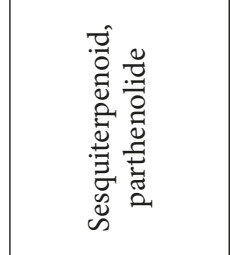 & 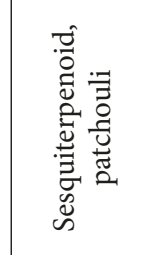 & 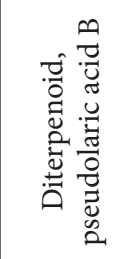 & 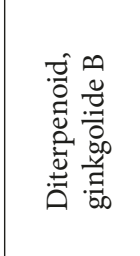 & 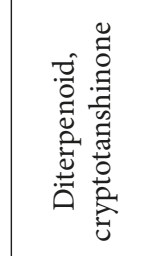 & 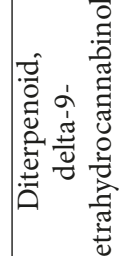 & 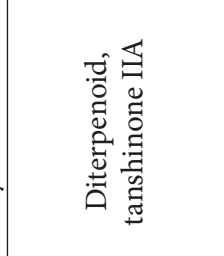 \\
\hline 胥 & 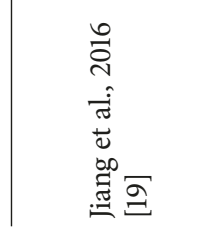 & 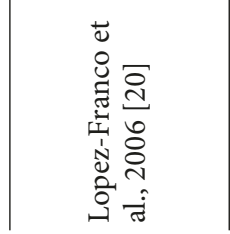 & 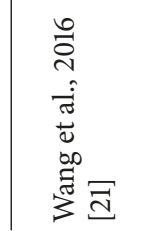 & 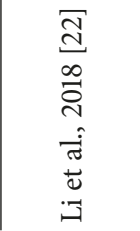 & 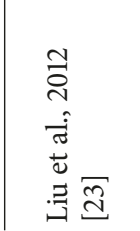 & 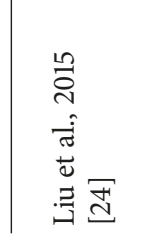 & 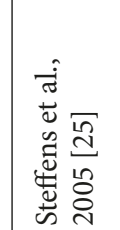 & 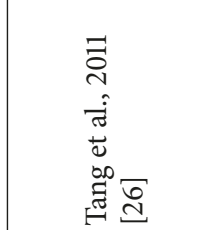 \\
\hline
\end{tabular}




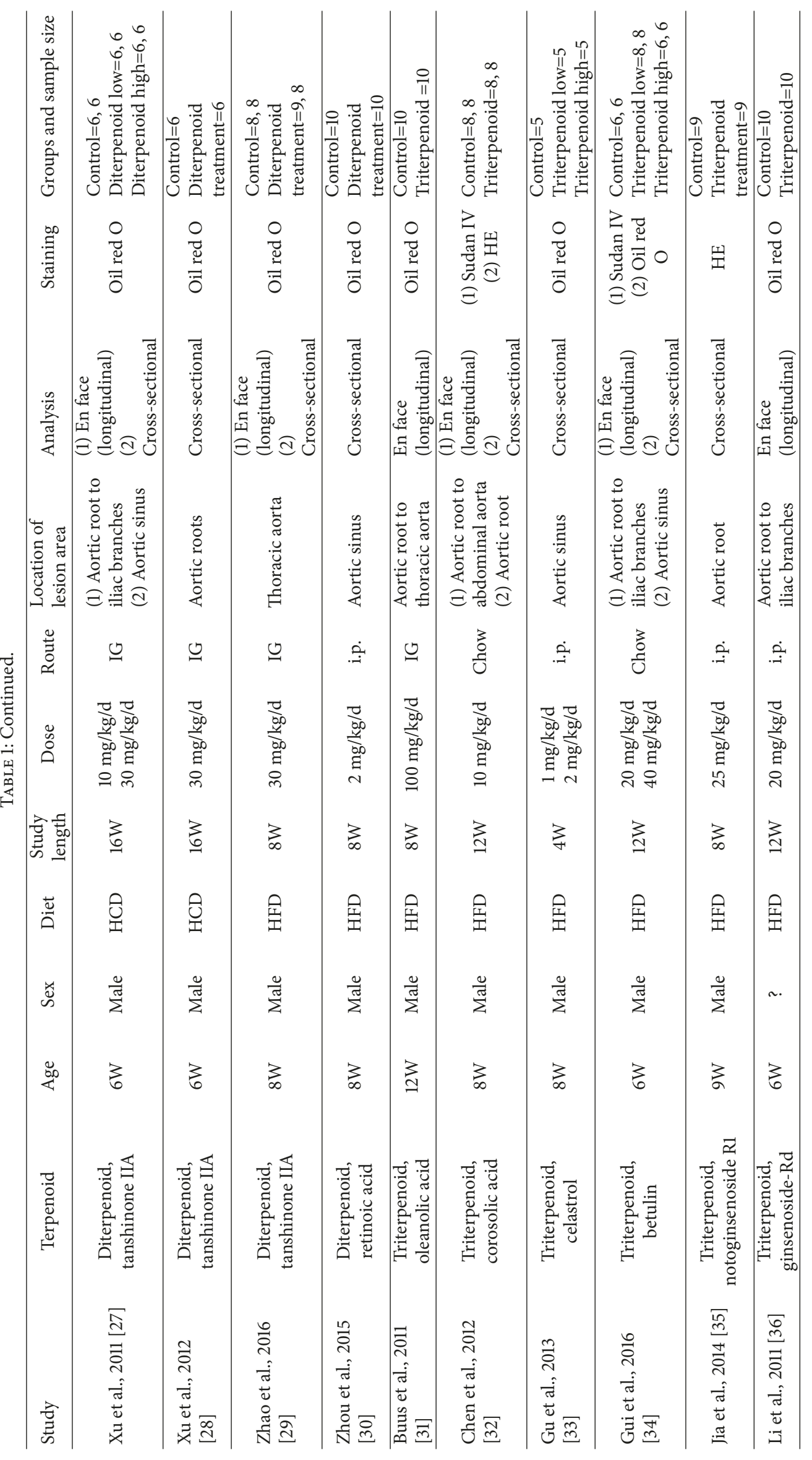




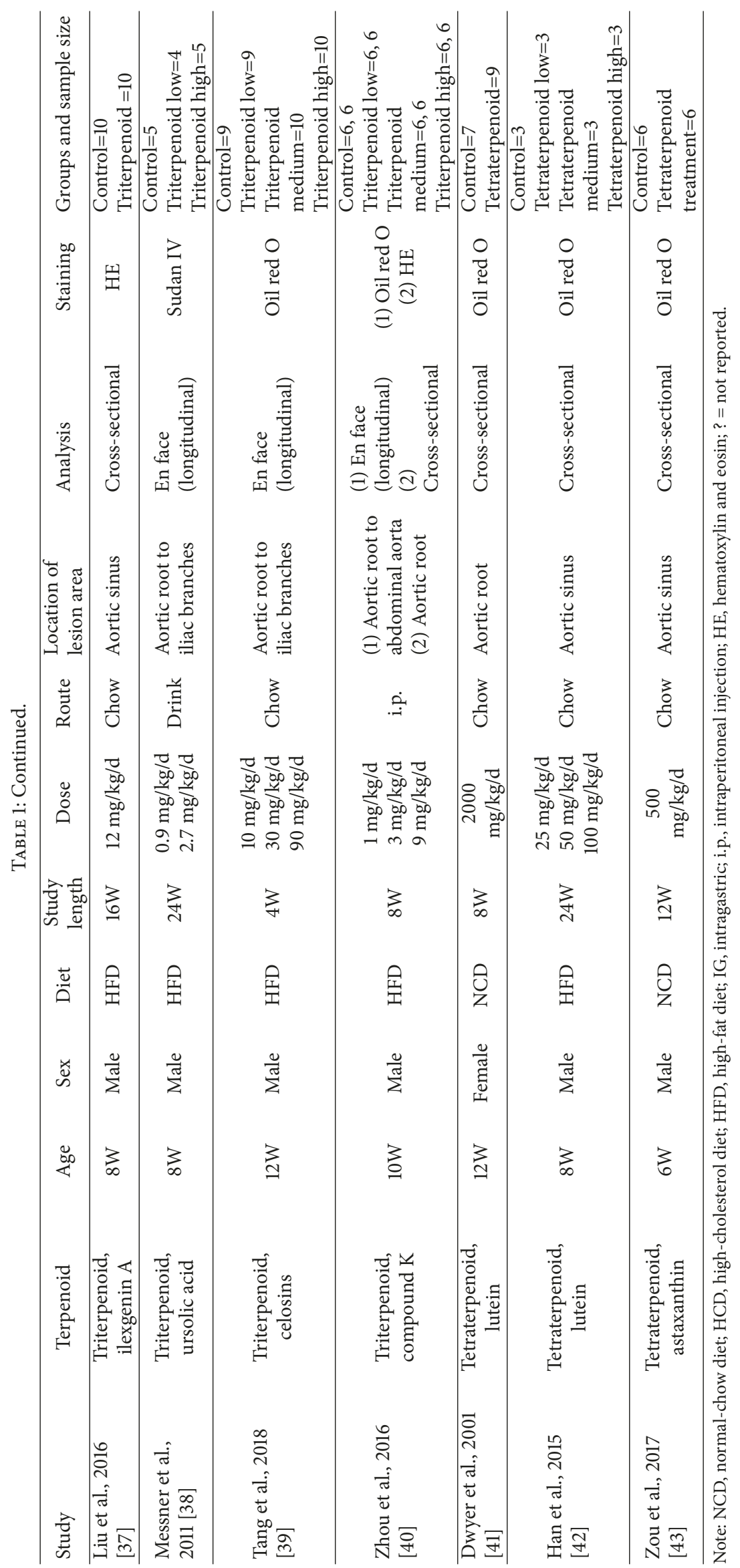




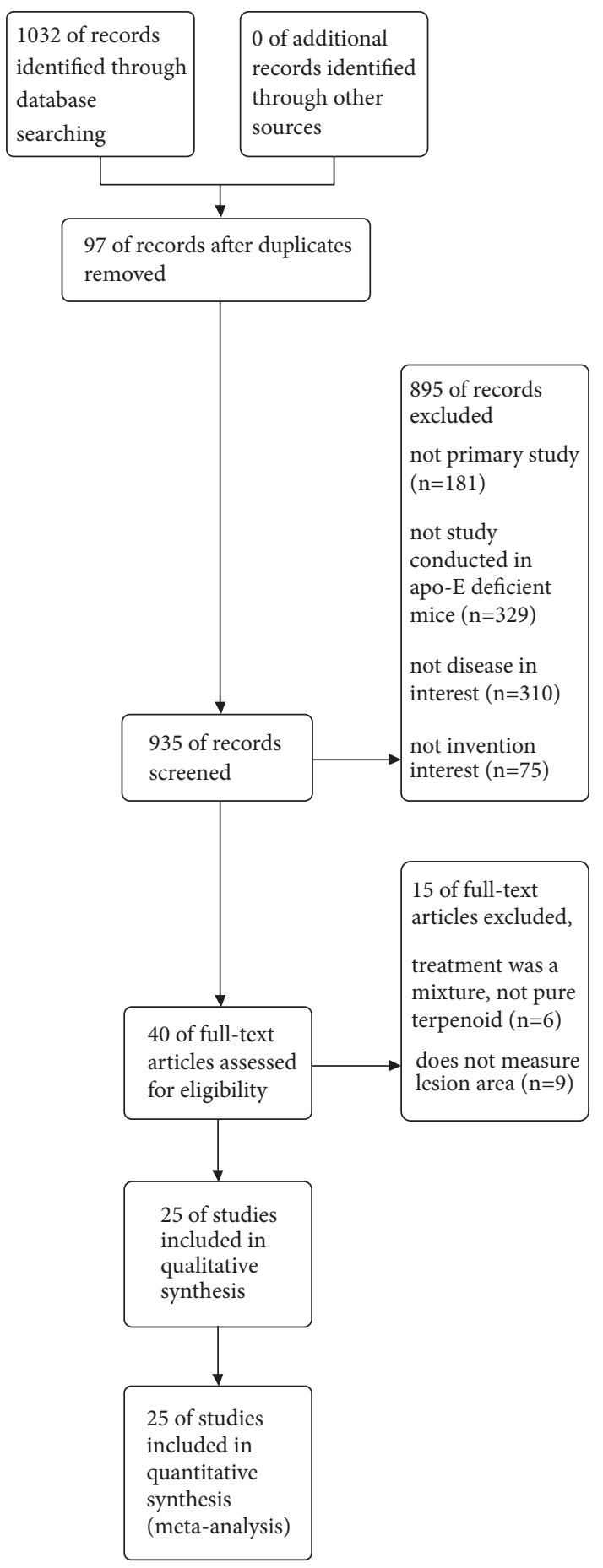

FIGURE 1: Flow diagram of the study identification and selection process.

Two studies used female animals [21, 41], 21 studies used male animals $[20,22-35,37-40,42,43]$, and the gender of the animals was not reported in two studies $[19,36]$.

Mice received normal chow diet in three studies [26, 41, 43], a high-cholesterol diet in two studies [27, 28], and a highfat diet in 20 studies [19-25, 29-40, 42].
Terpenoid administration was initiated in 4-week-old mice in one study [19], in 6-week-old mice in seven studies $[24,26-28,34,36,43]$, in 8-week-old mice in ten studies [21$23,29,30,32,33,37,38,42]$, in 9 -week-old mice in one study [35], in 10-week-old mice in two studies [25, 40], and in 12week-old mice in four studies [20,31, 39, 41].

The duration of terpenoid treatment varied from four weeks to 24 weeks.

Route of administration of terpenoid treatment was in drinking water in one study [38] and in the chow in seven studies [32, 34, 37, 39, 41-43], via an intragastric route in ten studies [21-29, 31] and via intraperitoneal injection in seven studies $[19,20,30,33,35,36,40]$.

Terpenoid doses varied among different studies. It mainly ranged from 1 to $100 \mathrm{mg} / \mathrm{kg} / \mathrm{d}$. In addition, 500 and 2000 $\mathrm{mg} / \mathrm{kg} / \mathrm{d}$ were used in two studies $[41,43]$.

All studies reported an aortic-root or -sinus lesion area. Furthermore, ten studies [20, 25, 27, 30, 33, 35, 37, 41-43] reported cross-sectional aortic lesion area, five studies [19, 31, $36,38,39$ ] reported longitudinal aortic lesion area (Table 1), and ten studies [21-24, 26, 28, 29, 32, 34, 40] reported both cross-sectional and longitudinal aortic lesion areas.

3.3. Quality Assessment. Assessment of study quality is presented in Figure 2. A total of 19 (73.1\%) studies were randomized, but the risks of bias due to allocation concealment and blinding were unclear. Sixteen studies had no missing outcomes data. The risk of selective outcomes reporting was unclear in nine studies. Across studies, the risk of bias from other sources was low.

Visual inspection of a funnel plot revealed substantial publication bias (Figure 3).

3.4. Effect of Terpenoids on Atherosclerotic Lesion Area. The effect of terpenoids on atherosclerotic lesion area was reported for 59 datasets obtained from 25 studies $(n=434$, ApoE -/- mice administered terpenoid; $n=273$, ApoE /- mice administered vehicle control). The meta-analysis demonstrated that overall terpenoids significantly reduced atherosclerotic lesion area when compared to vehicle control $(P<0.00001$; SMD: -0.55 ; 95\% CI: $-0.72,-0.39)$. There was no evidence of heterogeneity between studies $\left(I^{2}=0 \%\right.$, Figures 4 and 5).

Subgroup analyses were conducted to investigate the effects of terpenoid type and dose on atherosclerotic lesion area. In terpenoid type subgroup analyses, sesquiterpenoid ( $n=59$, ApoE -/- mice administered sesquiterpenoid; $n=38$, ApoE -/- mice administered vehicle control) significantly reduced atherosclerotic lesion area when compared to vehicle control ( $P=0.002$; SMD: -0.93 ; 95\% CI: $-1.52,-0.34)$; there was evidence of moderate heterogeneity between studies $\left(I^{2}=31 \%\right)$. Diterpenoid ( $n=184$, ApoE -/- mice administered diterpenoid; $n=121$, ApoE -/- mice administered vehicle control; $P=0.01$; SMD: -0.30 ; 95\% CI: $-0.54,-0.06)$, triterpenoid ( $n=167$, ApoE -/- mice administered triterpenoid; $n=98$, ApoE -/- mice administered vehicle control; $P<0.00001$; SMD: 0.66; 95\% CI: $-0.94,-0.39$ ), and tetraterpenoid ( $n=24$, ApoE -/- mice administered tetraterpenoid; $n=16$, ApoE -/- mice 


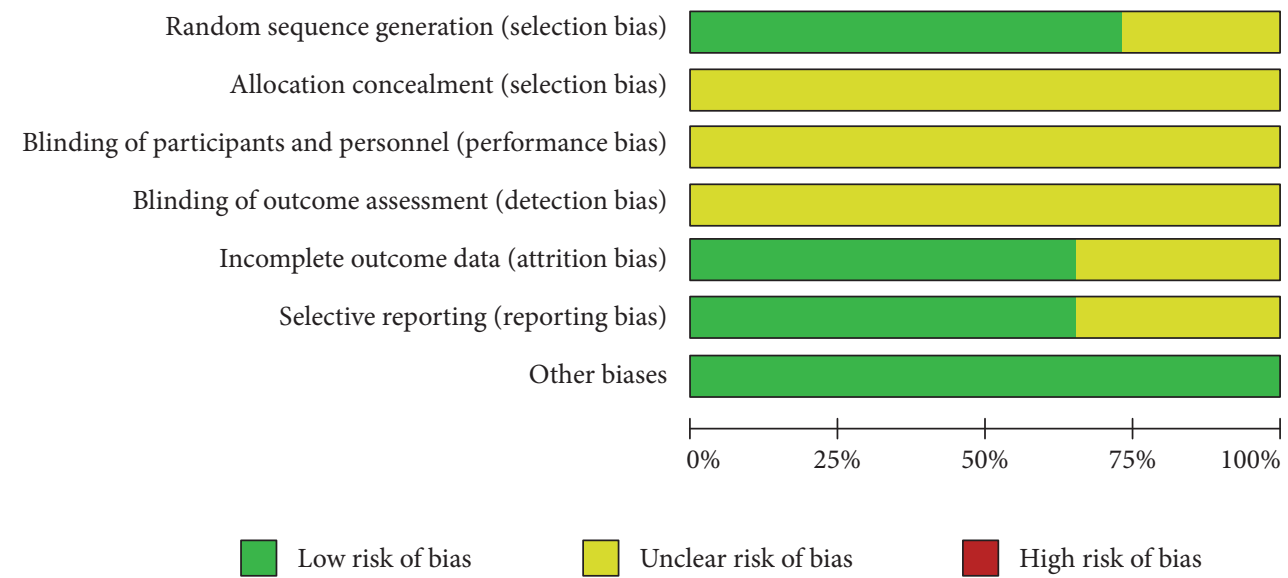

FIGURE 2: Risk of bias and quality assessment score (\%) for studies included in the meta-analysis.

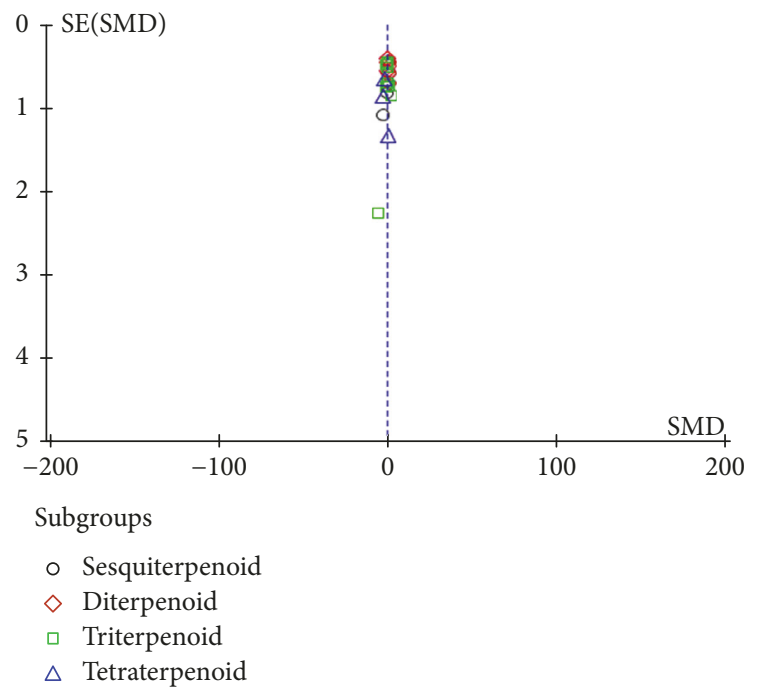

Figure 3: A funnel plot for evaluating publication bias.

administered vehicle control; $P<0.00001$; SMD: $-1.81 ; 95 \%$ CI: $-2.70,-0.91)$ significantly reduced atherosclerotic lesion area when compared to vehicle control; there was no evidence of heterogeneity between studies $\left(I^{2}=0 \%\right)$. In terpenoid dose subgroup analyses, high dose group $(n=58$, ApoE -/mice administered terpenoid $>50 \mathrm{mg} / \mathrm{kg} / \mathrm{d} ; n=35$, ApoE /- mice administered vehicle control) significantly reduced atherosclerotic lesion area when compared to vehicle control $(P=0.002$; SMD: -1.07 ; 95\% CI: $-1.74,-0.40)$; there was evidence of moderate heterogeneity between studies $\left(I^{2}=43 \%\right)$. Low dose group $(n=185$, ApoE -/- mice administered terpenoid $\leq 10 \mathrm{mg} / \mathrm{kg} / \mathrm{d} ; n=111$, ApoE -/- mice administered vehicle control; $P=0.0001$ SMD: $-0.51 ; 95 \% \mathrm{CI}:-0.76,-0.25)$ and medium dose group $(n=191$, ApoE - $/$ - mice administered terpenoid $>10 \mathrm{mg} / \mathrm{kg} / \mathrm{d}, \leq 50 \mathrm{mg} / \mathrm{kg} / \mathrm{d} ; n=127$, ApoE -/- mice administered vehicle control; $P<0.0001$; SMD: -0.48 ; $95 \% \mathrm{CI}$ : $-0.72,-0.24)$ significantly reduced atherosclerotic lesion area when compared to vehicle control; there was no evidence of heterogeneity between studies $\left(I^{2}=0 \%\right)$.
Sensitivity analysis that substituted the fixed effect model for the random effects model did not change the overall findings (SMD - $0.55(-0.72,-0.39)$ vs. $-0.58(-0.69,-0.46)$ and SMD $-0.55(-0.72,-0.39)$ vs. $-0.57(-0.68,-0.45))$.

\section{Discussion}

The use of animal models provides a valuable approach to preclinical research, which informs treatment strategies for human diseases. Previous evidence from experiments in animals suggests that natural terpenoids have potential benefits for the treatment of atherosclerosis. However, parameters such as type and age of animal, sample size, housing conditions, and length of follow-up vary across studies. A synthesis and quantitative analysis of the data from animal models that accounts for these sources of heterogeneity may provide insight into the benefits of terpenoids as clinically desirable therapeutic agents in atherosclerosis. Therefore, we performed this systematic review and meta-analysis to evaluate the effects terpenoid administration on atherosclerotic lesion area in ApoE -/- mice. Findings showed that terpenoid administration significantly reduced aortic atherosclerosis lesion area compared to vehicle control.

The terpenoid family constitutes several members, including hemiterpenoid, monoterpenoid, sesquiterpenoid, diterpenoid, sesterterpenoid, triterpenoid, tetraterpenoid, and polyterpenoid [60]. In subanalyses stratified by number of isoprene groups, sesquiterpenoid, diterpenoid, triterpenoid, and tetraterpenoid significantly reduced aortic atherosclerosis lesion area compared to vehicle control in ApoE-/- mice.

To the authors' knowledge, this systematic review and meta-analysis is the first to evaluate the effects of terpenoids on atherosclerosis in ApoE -/- mice. Findings are expected to provide a scientific basis for clinical trials of terpenoids in cardiovascular diseases.

There was no heterogeneity between studies in the overall analysis, but there was a moderate degree of heterogeneity between studies in the analysis of sesquiterpenoid and high dose group. Potential sources of heterogeneity include age 


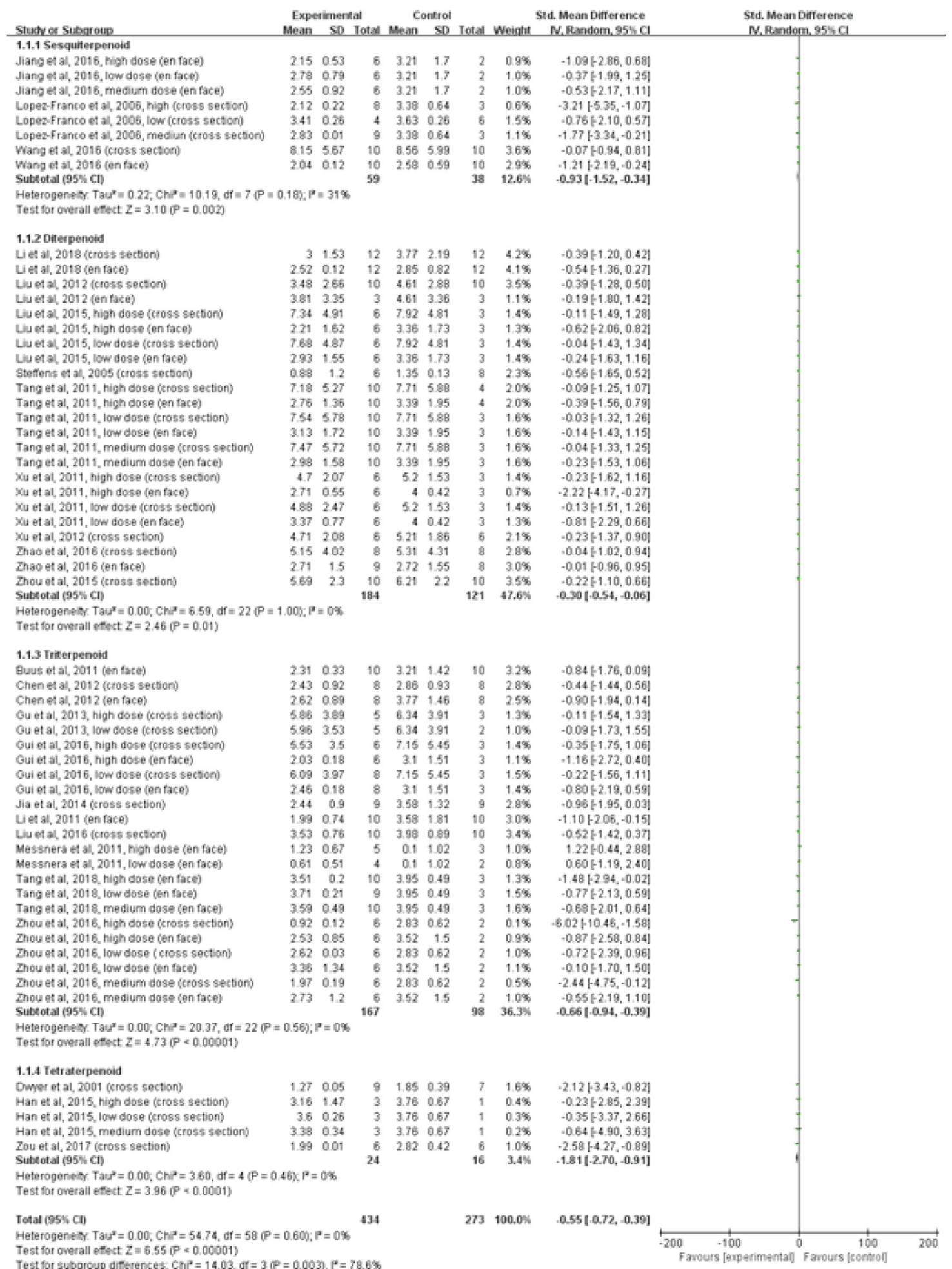

FIGURE 4: A forest plot of the effects of different terpenoids types on atherosclerotic lesion area. Subgroup analyses evaluated the effects of sesquiterpenoid, diterpenoid, triterpenoid, and tetraterpenoid. SD, standard deviation; CI, confidence interval; Std, standard; IV, inverse variance. 


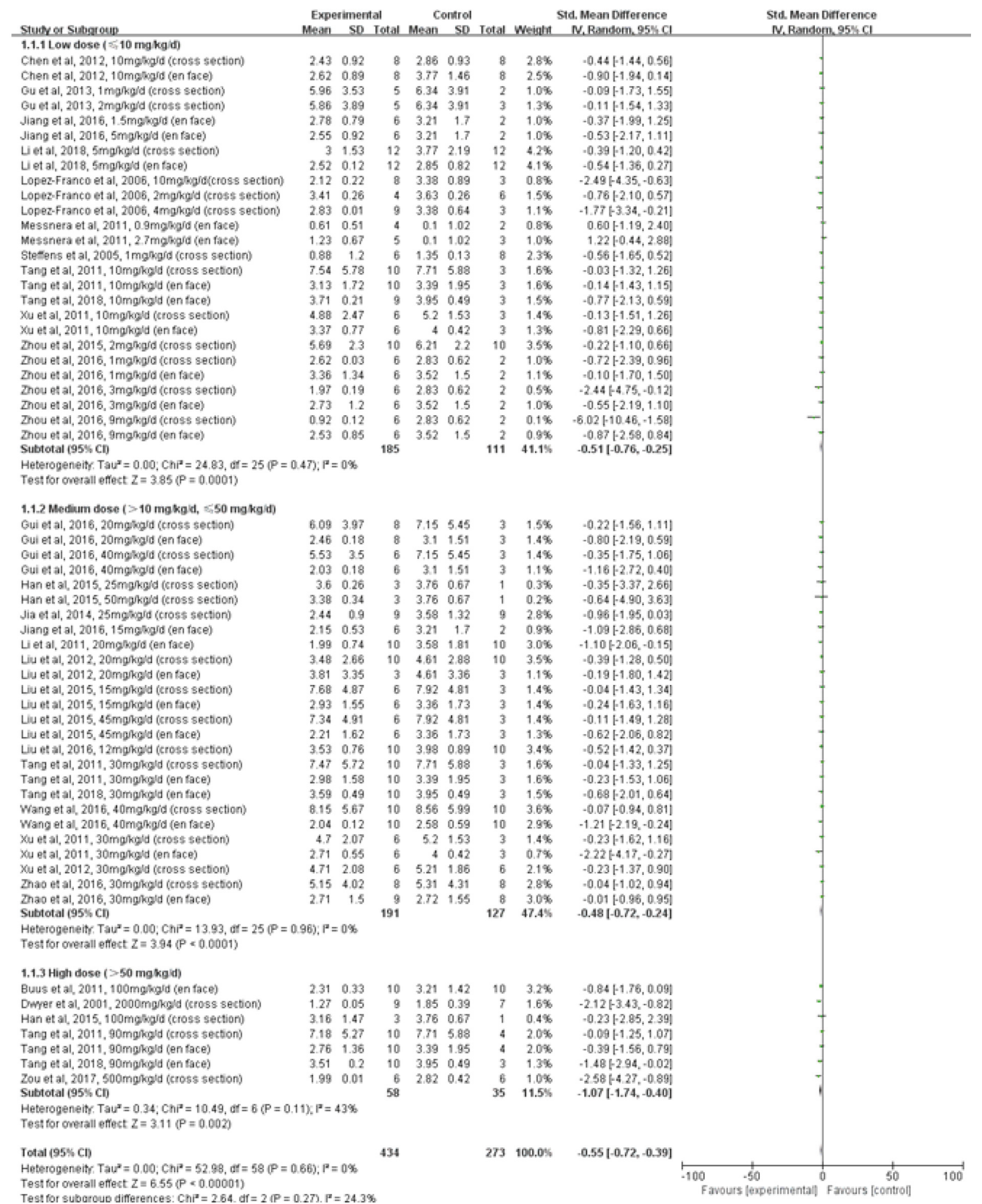

FIGURE 5: A forest plot of the effects of different terpenoids doses on atherosclerotic lesion area. Subgroup analyses evaluated the effects of low dose ( $\leq 10 \mathrm{mg} / \mathrm{kg} / \mathrm{d})$, medium dose $(>10 \mathrm{mg} / \mathrm{kg} / \mathrm{d}, \leq 50 \mathrm{mg} / \mathrm{kg} / \mathrm{d})$, and high dose ( $>50 \mathrm{mg} / \mathrm{kg} / \mathrm{d})$. SD, standard deviation; CI, confidence interval; Std, standard; IV, inverse variance. 
and sex of mice and diet administered, each of which can influence the progression of atherosclerosis $[1,61]$. Furthermore, method of measurement can affect the assessment. Ten studies reported cross-sectional aortic lesion area, five studies reported longitudinal aortic lesion area, and ten studies reported both cross-sectional and longitudinal aortic lesion area.

Study Limitations. This meta-analysis was associated with several limitations. First, the relevance of our findings to humans is limited by species specific differences in lipoprotein metabolism and vascular physiology [62]. The ApoE /- mouse model is well established for studying atherosclerosis, and the principal characteristics and progression of atherosclerosis in ApoE -/- mice and human subjects appear similar $[15,16]$; however, there are differences in pathogenesis. Specifically, the location of the atherosclerotic plaque may differ due to variations in heart rate, blood pressure, and hemodynamics. Atherosclerotic plaque builds up in the root of the aorta and in the brachiocephalic artery in ApoE /- mice and the coronary artery, the carotid artery, the iliac artery, and the arteries of the lower limb in humans. Second, age is a risk factor in progression of atherosclerosis; therefore, due to differences in life cycle, the natural history of atherosclerotic disease in mice cannot be directly translated to humans. Third, patients usually present to the clinic with advanced atherosclerosis, but the included studies administered terpenoids to mice before disease had progressed and were therefore evaluating early prevention rather than benefit of treatment in advanced disease. Fourth, other animal models of atherosclerosis, such as LDLR -/- mice, rabbits, and hamsters, were not included in this meta-analysis. Fifth, only one parameter, atherosclerotic lesion area, was used to evaluate the effects of terpenoids on atherosclerosis. Other parameters, such as low density lipoprotein (LDL), high density lipoprotein (HDL), total cholesterol (TC), triglycerides (TG), and body mass index (BMI), were not considered. [63] Sixth, the sample sizes of some included studies were relatively small. Seventh, hemiterpenoid, monoterpenoid, and polyterpenoid were not assessed in the analysis. Further investigations in animal models using larger sample sizes are warranted to determine if terpenoids are beneficial for the treatment of atherosclerosis in humans.

The present meta-analysis revealed that terpenoid administration is effective for attenuating aortic atherosclerosis in ApoE-/- mice. In particular, sesquiterpenoid, diterpenoid, triterpenoid, and tetraterpenoid have a potential therapeutic effect with a wide range of doses. Large scale, prospective, and well-designed animal studies are needed to enhance our knowledge of the mechanism of terpenoids for the treatment of atherosclerosis. Randomized controlled trails in humans are required to confirm that terpenoids have clinical benefit as antiatherosclerotic agents.

\section{Data Availability}

The data used and/or analyzed during the current study are available from the corresponding author on reasonable request.

\section{Conflicts of Interest}

The authors declare that they have no conflicts of interest.

\section{Authors' Contributions}

Han Liu conducted the analysis and wrote the manuscript; Yang Zhang and Siqiao Sun collected and performed a preliminary analysis of references; Shuai Wang revised the manuscript and approved it for submission.

\section{References}

[1] D. Mozaffarian, J. Benjamin, S. Go et al., "Heart disease and stroke statistics-2015 update: a report from the American Heart Association," Circulation, vol. 131, no. 4, pp. 29-322, 2015.

[2] Z. Yang, J. Liu, J. Ge, L. Chen, Z. Zhao, and W. Yang, "Prevalence of cardiovascular disease risk factor in the Chinese population: the 2007-2008 China National Diabetes and Metabolic Disorders Study, European Heart Journal, vol. 33, no. 2, pp. 213-220, 2012.

[3] V. M. Victor, M. Rocha, E. Solá, C. Bañuls, K. GarciaMalpartida, and A. Hernández-Mijares, "Oxidative stress, endothelial dysfunction and atherosclerosis," Current Pharmaceutical Design, vol. 15, no. 26, pp. 2988-3002, 2009.

[4] S. Koba and T. Hirano, "Dyslipidemia and atherosclerosis," Nihon Rinsho, vol. 69, pp. 138-143, 2011.

[5] J. Logue, H. M. Murray, P. Welsh et al., "Obesity is associated with fatal coronary heart disease independently of traditional risk factors and deprivation," Heart, vol. 97, no. 7, pp. 564-568, 2011.

[6] T. J. Ford, D. Corcoran, and C. Berry, "Stable coronary syndromes: Pathophysiology, diagnostic advances and therapeutic need," Heart, vol. 104, no. 4, pp. 284-292, 2018.

[7] A. Pirillo and A. L. Catapano, "Berberine, a plant alkaloid with lipid- and glucose-lowering properties: from in vitro evidence to clinical studies," Atherosclerosis, vol. 243, no. 2, pp. 449-461, 2015.

[8] L. Xiao, L. Liu, X. Guo et al., "Quercetin attenuates high fat dietinduced atherosclerosis in apolipoprotein E knockout mice: A critical role of NADPH oxidase," Food and Chemical Toxicology, vol. 105, pp. 22-33, 2017.

[9] X. Zhang, M. Liu, L. Qiao et al., "Ginsenoside Rb1 enhances atherosclerotic plaque stability by skewing macrophages to the M2 phenotype," Journal of Cellular and Molecular Medicine, vol. 22, no. 1, pp. 409-416, 2018.

[10] L. Cao, X. Zhang, F. Cao et al., "Inhibiting inducible miR-223 further reduces viable cells in human cancer cell lines MCF-7 and PC3 treated by celastrol," BMC Cancer, vol. 15, no. 1, article $873,2015$.

[11] R. Gallily, Z. Yekhtin, and L. O. Hanuš, “The anti-inflammatory properties of terpenoids from cannabis," Cannabis and Cannabinoid Research, vol. 3, no. 1, pp. 282-290, 2018.

[12] Z. Shi, Y. Chen, C. Lu et al., "Resolving neuroinflammation, the therapeutic potential of the anti-malaria drug family of artemisinin," Pharmacological Research, vol. 136, pp. 172-180, 2018.

[13] M. Brodmann, K. Keirse, D. Scheinert et al., "Drug-coated balloon treatment for femoropopliteal artery disease: the IN.PACT global study de novo in-stent restenosis imaging cohort," JACC: Cardiovascular Interventions, vol. 10, no. 20, pp. 2113-2123, 2017. 
[14] F. L. Gomes, R. C. Maranhão, E. R. Tavares et al., "Regression of atherosclerotic plaques of cholesterol-fed rabbits by combined chemotherapy with paclitaxel and methotrexate carried in lipid core nanoparticles," Journal of Cardiovascular Pharmacology and Therapeutics, vol. 23, no. 6, pp. 561-569, 2018.

[15] J. Jawien, "The role of an experimental model of atherosclerosis: apoE-knockout mice in developing new drugs against atherogenesis," Current Pharmaceutical Biotechnology, vol. 13, no. 13, pp. 2435-2439, 2012.

[16] E. C. Vasquez, V. A. Peotta, A. L. Gava, T. M. Pereira, and S. S. Meyrelles, "Cardiac and vascular phenotypes in the apolipoprotein E-deficient mouse," Journal of Biomedical Science, vol. 19, no. 1, p. 22, 2012.

[17] R. de Vries, C. Hooijmans, M. Langendam et al., "A protocol format for the preparation, registration and publication of systematic reviews of animal intervention studies," Evidencebased Preclinical Medicine, vol. 2, pp. 1-9, 2015.

[18] C. R. Hooijmans and M. Ritskes-Hoitinga, "Progress in using systematic reviews of animal studies to improve translational research," PLoS Medicine, vol. 10, no. 7, Article ID e1001482, 2013.

[19] W. Jiang, Y. Cen, Y. Song et al., "Artesunate attenuated progression of atherosclerosis lesion formation alone or combined with rosuvastatin through inhibition of pro-inflammatory cytokines and pro-inflammatory chemokines," Phytomedicine, vol. 23, no. 11, pp. 1259-1266, 2016.

[20] O. López-Franco, P. Hernández-Vargas, G. Ortiz-Muñoz et al., "Parthenolide modulates the NF-kappaB-mediated inflammatory responses in experimental atherosclerosis," Arteriosclerosis, Thrombosis and Vascular Biology, vol. 26, no. 8, pp. 1864-1870, 2006.

[21] H.-T. Wang, Z.-Z. Wang, Z.-C. Wang et al., "Patchouli alcohol attenuates experimental atherosclerosis via inhibiting macrophage infiltration and its inflammatory responses," Biomedicine \& Pharmacotherapy, vol. 83, pp. 930-935, 2016.

[22] T. Li, W. Wang, Y. Li et al., "Pseudolaric acid B attenuates atherosclerosis progression and inflammation by suppressing PPAR $\gamma$-mediated NF- $\kappa \mathrm{B}$ activation," International Immunopharmacology, vol. 59, pp. 76-85, 2018.

[23] X. Liu, G. Zhao, Y. Yan et al., "Ginkgolide B reduces atherogenesis and vascular inflammation in $\mathrm{ApoE}^{-/-}$mice," PLoS ONE, vol. 7, no. 5, Article ID e36237, 2012.

[24] Z. Liu, S. Xu, X. Huang et al., "Cryptotanshinone, an orally bioactive herbal compound from Danshen, attenuates atherosclerosis in apolipoprotein E-deficient mice: role of lectin-like oxidized LDL receptor-1 (LOX-1)," British Journal of Pharmacology, vol. 172, no. 23, pp. 5661-5675, 2015.

[25] S. Steffens, N. R. Veillard, C. Arnaud et al., "Low dose oral cannabinoid therapy reduces progression of atherosclerosis in mice," Nature, vol. 434, no. 7034, pp. 782-786, 2005.

[26] F.-T. Tang, Y. Cao, T.-Q. Wang et al., “Tanshinone IIA attenuates atherosclerosis in $\mathrm{ApoE}^{-1-}$ mice through down-regulation of scavenger receptor expression," European Journal of Pharmacology, vol. 650, no. 1, pp. 275-284, 2011.

[27] S. Xu, Z. Liu, Y. Huang et al., "Tanshinone II-A inhibits oxidized LDL-induced LOX-1 expression in macrophages by reducing intracellular superoxide radical generation and NF- $\kappa \mathrm{B}$ activation," Translational Research, vol. 160, no. 2, pp. 114-124, 2012.

[28] S. Xu, P. J. Little, T. Lan et al., "Tanshinone II-A attenuates and stabilizes atherosclerotic plaques in apolipoprotein-E knockout mice fed a high cholesterol diet," Archives of Biochemistry and Biophysics, vol. 515, no. 1-2, pp. 72-79, 2011.

[29] D. Zhao, L. Tong, L. Zhang, H. Li, Y. Wan, and T. Zhang, "Tanshinone II A stabilizes vulnerable plaques by suppressing RAGE signaling and NF- $\kappa$ B activation in apolipoprotein-Edeficient mice," Molecular Medicine Reports, vol. 14, no. 6, pp. 4983-4990, 2016.

[30] W. Zhou, J. Lin, H. Chen, J. Wang, Y. Liu, and M. Xia, "Retinoic acid induces macrophage cholesterol efflux and inhibits atherosclerotic plaque formation in apoE-deficient mice," British Journal of Nutrition, vol. 114, no. 4, pp. 509-518, 2015.

[31] N. H. Buus, N. C. Hansson, R. Rodriguez-Rodriguez, E. Stankevicius, M. R. Andersen, and U. Simonsen, "Antiatherogenic effects of oleanolic acid in apolipoprotein E knockout mice," European Journal of Pharmacology, vol. 670, no. 2-3, pp. 519526, 2011.

[32] H. Chen, J. Yang, Q. Zhang, L. Chen, and Q. Wang, "Corosolic acid ameliorates atherosclerosis in apolipoprotein E-deficient mice by regulating the nuclear factor- $\kappa \mathrm{B}$ signaling pathway and inhibiting monocyte chemoattractant protein-1 expression," Circulation Journal, vol. 76, no. 4, pp. 995-1003, 2012.

[33] L. Gu, W. Bai, S. Li et al., "Celastrol prevents atherosclerosis via inhibiting LOX-1 and oxidative stress," PLoS ONE, vol. 8, no. 6, Article ID e65477, 2013.

[34] Y.-Z. Gui, H. Yan, F. Gao, C. Xi, H.-H. Li, and Y.-P. Wang, "Betulin attenuates atherosclerosis in apoE-/-mice by upregulating ABCA1 and ABCG1," Acta Pharmacologica Sinica, vol. 37, no. 10, pp. 1337-1348, 2016.

[35] C. Jia, M. Xiong, P. Wang et al., "Notoginsenoside R1 attenuates atherosclerotic lesions in ApoE deficient mouse model," PLoS ONE, vol. 9, no. 6, Article ID e99849, 2014.

[36] J. Li, Z. Xie, Y. Tang, J. Zhou, and Y. Guan, "Ginsenoside$\mathrm{Rd}$, a purified component from panax notoginseng saponins, prevents atherosclerosis in apoE knockout mice," European Journal of Pharmacology, vol. 652, no. 1-3, pp. 104-110, 2011.

[37] C. Liu, J. Zhao, Y. Liu et al., "A novel pentacyclic triterpenoid, Ilexgenin A, shows reduction of atherosclerosis in apolipoprotein E deficient mice," International Immunopharmacology, vol. 40, pp. 115-124, 2016.

[38] B. Messner, I. Zeller, C. Ploner et al., "Ursolic acid causes DNAdamage, P53-mediated, mitochondria- and caspase-dependent human endothelial cell apoptosis, and accelerates atherosclerotic plaque formation in vivo," Atherosclerosis, vol. 219, no. 2, pp. 402-408, 2011.

[39] Y. Tang, H. Wu, B. Shao, Y. Wang, C. Liu, and M. Guo, “Celosins inhibit atherosclerosis in ApoE -/- mice and promote autophagy flow," Journal of Ethnopharmacology, vol. 215, pp. 74-82, 2017.

[40] L. Zhou, Y. Zheng, Z. Li et al., "Compound K attenuates the development of atherosclerosis in ApoE(-/-) mice via LXR $\alpha$ activation," International Journal of Molecular Sciences, vol. 17, no. 7, Article ID E1054, 2016.

[41] J. H. Dwyer, M. Navab, K. M. Dwyer et al., "Oxygenated carotenoid lutein and progression of early atherosclerosis: the Los Angeles atherosclerosis study," Circulation, vol. 103, no. 24, pp. 2922-2927, 2001.

[42] H. Han, W. Cui, L. Wang et al., "Lutein prevents high fat dietinduced atherosclerosis in ApoE-deficient mice by inhibiting NADPH oxidase and increasing PPAR expression," Lipids, vol. 50, no. 3, pp. 261-273, 2015.

[43] T. Zou, S. Zhu, F. Luo et al., "Effects of astaxanthin on reverse cholesterol transport and atherosclerosis in mice," BioMed 
Research International, vol. 2017, Article ID 4625932, 6 pages, 2017.

[44] C. R. Hooijmans, M. M. Rovers, R. B. M. De Vries, M. Leenaars, M. Ritskes-Hoitinga, and M. W. Langendam, "SYRCLE's risk of bias tool for animal studies," BMC Medical Research Methodology, vol. 14, no. 1, article no. 43, 2014.

[45] Y. Bao, L. Wang, Y. Xu et al., "Salvianolic acid B inhibits macrophage uptake of modified low density lipoprotein (mLDL) in a scavenger receptor CD36-dependent manner," Atherosclerosis, vol. 223, no. 1, pp. 152-159, 2012.

[46] S. Bechor, N. Zolberg Relevy, A. Harari et al., "9-cis $\beta$-carotene increased cholesterol efflux to HDL in macrophages," Nutrients, vol. 8, no. 7, article 435, 2016.

[47] P. Fernández-Robredo, L. M. Sádaba, A. Salinas-Alamán, S. Recalde, J. A. Rodríguez, and A. García-Layana, "Effect of lutein and antioxidant supplementation on VEGF expression, MMP2 activity, and ultrastructural alterations in apolipoprotein Edeficient mouse," Oxidative Medicine and Cellular Longevity, vol. 2013, Article ID 213505, 11 pages, 2013.

[48] A. J. Guri, S. A. Misyak, R. Hontecillas et al., "Abscisic acid ameliorates atherosclerosis by suppressing macrophage and CD4+ T cell recruitment into the aortic wall," The Journal of Nutritional Biochemistry, vol. 21, no. 12, pp. 1178-1185, 2010.

[49] T. H.-W. Huang, V. Razmovski-Naumovski, N. K. Salam et al., "A novel LXR- $\alpha$ activator identified from the natural product Gynostemma pentaphyllum," Biochemical Pharmacology, vol. 70, no. 9, pp. 1298-1308, 2005.

[50] A. Katsumata, M. Kimura, H. Saigo et al., "Changes in esculeoside a content in different regions of the tomato fruit during maturation and heat processing," Journal of Agricultural and Food Chemistry, vol. 59, no. 8, pp. 4104-4110, 2011.

[51] Q. Li, W. Zhao, X. Zeng, and Z. Hao, "Ursolic acid attenuates atherosclerosis in ApoE-/- mice: role of LOX-1 mediated by ROS/NF- $\kappa$ B pathway," Molecules, vol. 23, no. 5, Article ID E1101, 2018.

[52] X. Liu, C. Y. Guo, X. J. Ma et al., "Anti-inflammatory effects of tanshinone IIA on atherosclerostic vessels of ovariectomized $\mathrm{ApoE}^{-1-}$ mice are mediated by estrogen receptor activation and through the ERK signaling pathway," Cellular Physiology and Biochemistry, vol. 35, no. 5, pp. 1744-1755, 2015.

[53] J. J. Yoon, Y. J. Lee, B. H. Han et al., "Protective effect of betulinic acid on early atherosclerosis in diabetic apolipoprotein-E gene knockout mice," European Journal of Pharmacology, vol. 796, pp. 224-232, 2017.

[54] A. Harari, R. Abecassis, N. Relevi et al., "Prevention of atherosclerosis progression by 9 -cis- $\beta$-carotene rich alga dunaliella in apoE-deficient mice," BioMed Research International, vol. 2013, Article ID 169517, 7 pages, 2013.

[55] G. Liu, B. Wang, J. Zhang, H. Jiang, and F. Liu, “Total panax notoginsenosides prevent atherosclerosis in apolipoprotein Eknockout mice: role of downregulation of CD40 and MMP-9 expression," Journal of Ethnopharmacology, vol. 126, no. 2, pp. 350-354, 2009.

[56] L. Liu, P. Liao, B. Wang, X. Fang, W. Li, and S. Guan, "Oral administration of baicalin and geniposide induces regression of atherosclerosis via inhibiting dendritic cells in ApoE-knockout mice," International Immunopharmacology, vol. 20, no. 1, pp. 197-204, 2014.

[57] N. Relevy, D. Harats, A. Harari et al., "Vitamin A-deficient diet accelerated atherogenesis in apolipoprotein $\mathrm{E}^{-/-}$mice and dietary $\beta$-carotene prevents this consequence," BioMed
Research International, vol. 2015, Article ID 758723, 9 pages, 2015.

[58] N. Takeda, I. Manabe, T. Shindo et al., "Synthetic retinoid Am80 reduces scavenger receptor expression and atherosclerosis in mice by inhibiting IL-6," Arteriosclerosis, Thrombosis, and Vascular Biology, vol. 26, no. 5, pp. 1177-1183, 2006.

[59] G. Zhao, S. Tang, Y. Lv et al., "Antagonism of betulinic acid on LPS-mediated inhibition of ABCA1 and cholesterol efflux through inhibiting nuclear factor-kappab signaling pathway and miR-33 expression," PLoS ONE, vol. 8, no. 9, p. e74782, 2013.

[60] D. W. Christianson, "Structural and chemical biology of terpenoid cyclases," Chemical Reviews, vol. 117, no. 17, pp. 1157011648, 2017.

[61] M. Liu, W. Zhang, X. Li, J. Han, Y. Chen, and Y. Duan, "Impact of age and sex on the development of atherosclerosis and expression of the related genes in apoE deficient mice," Biochemical and Biophysical Research Communications, vol. 469, no. 3, pp. 456-462, 2016.

[62] S. Zadelaar, R. Kleemann, L. Verschuren et al., "Mouse models for atherosclerosis and pharmaceutical modifiers," Arteriosclerosis, Thrombosis, and Vascular Biology, vol. 27, no. 8, pp. 17061721, 2007.

[63] A. Zmysłowski and A. Szterk, "Current knowledge on the mechanism of atherosclerosis and pro-atherosclerotic properties of oxysterols," Lipids in Health and Disease, vol. 16, no. 1, article $188,2017$. 

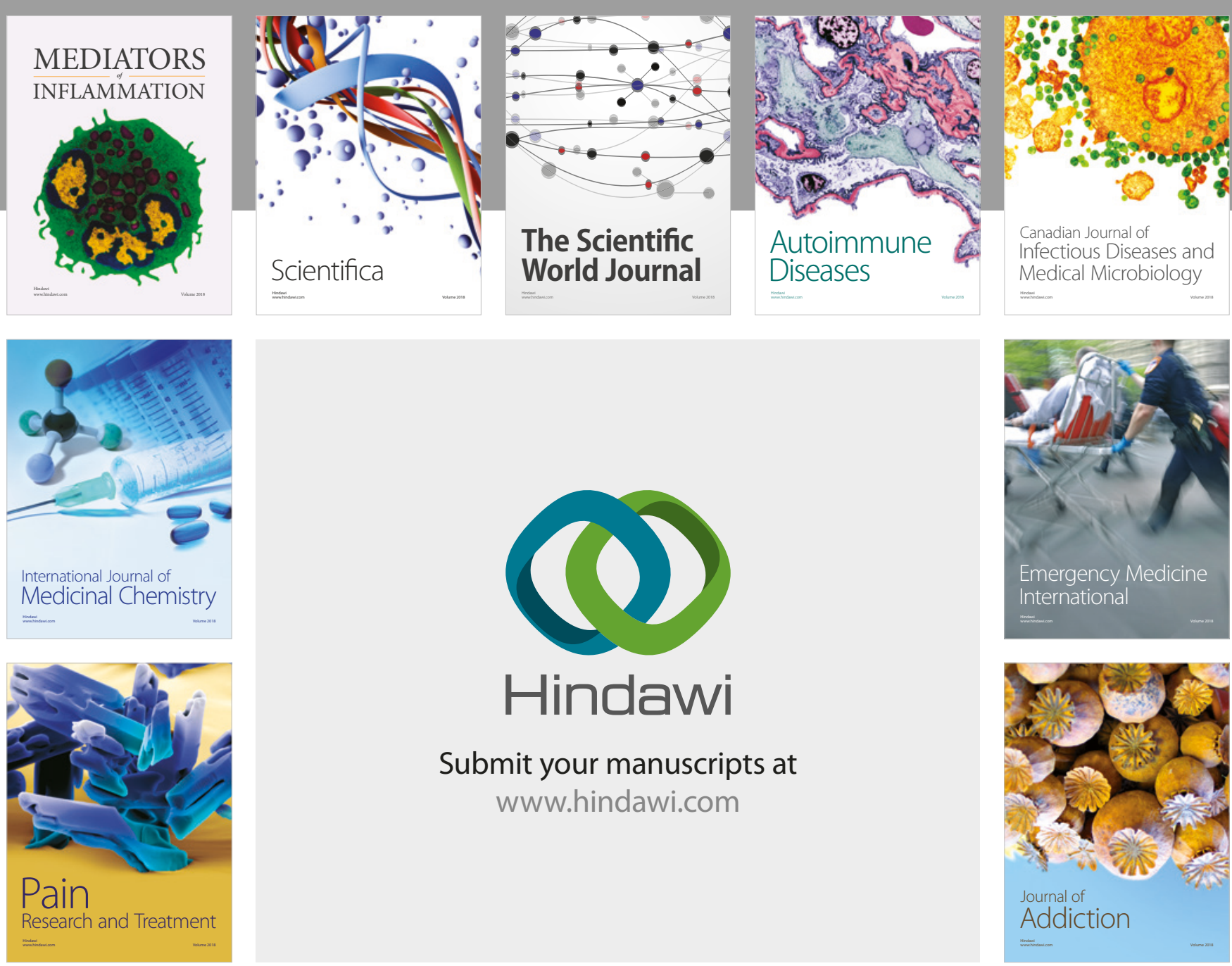

Canadian Journal of
Infectious Diseases and Medical Microbiology

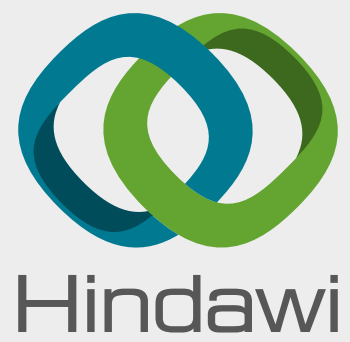

Submit your manuscripts at

www.hindawi.com
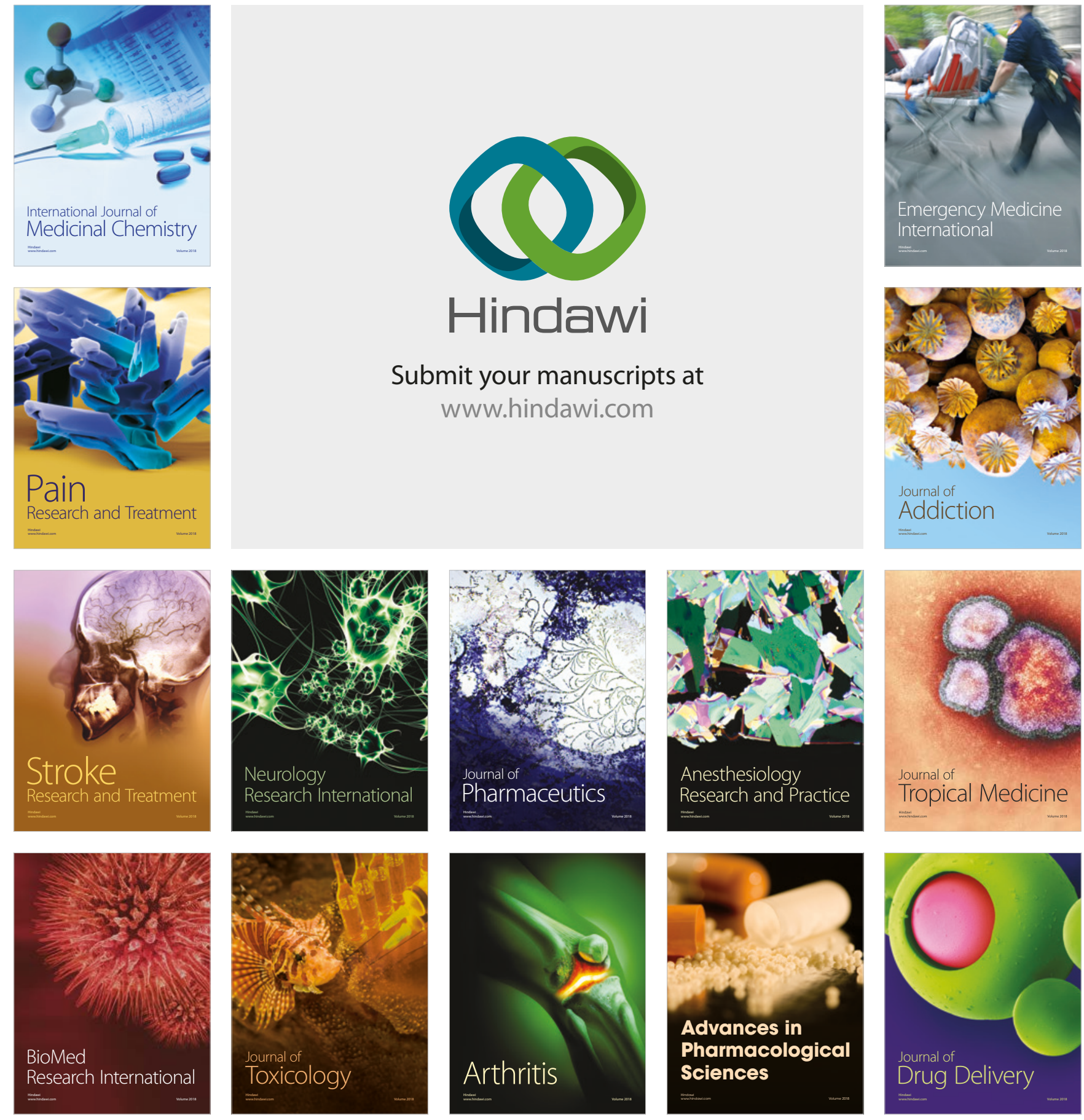